week titration period, provided a consistent beneficial outcome across doses. Here we describe the titration and dosing of selexipag in a real-world clinical setting, using EXPOSURE study data.

EXPOSURE (EUPAS19085) is an ongoing, international, multicentre, prospective, observational study of $\mathrm{PAH}$ patients initiating any new PAH-specific therapy. These analyses include all patients receiving selexipag with follow-up data. Titration was considered complete once a stable dose was taken for $\geq 3$ weeks after the highest identified dose. Patients entered the maintenance phase on week 2 of that stable dose. Titration and maintenance phases were defined after data collection. Patient characteristics and treatment patterns at selexipag initiation are described. Values are median (range) unless otherwise stated.

As of June 2020, 300 selexipag-treated patients had followup data. At selexipag initiation: age and time from $\mathrm{PAH}$ diagnosis was $60(18-87)$ and $2.7(0.0-51.3)$ years; 70\% patients were female and $60 \%$ were at intermediate risk of 1-year mortality (COMPERA approach). PAH was classified as idiopathic (51.3\%), associated (41.7\%), drug/toxin induced (2.7\%), and heritable (4.3\%). Exposure to selexipag was 5.4 (0.2-29.0) months. Most (77\%) patients initiated selexipag as part of a triple combination therapy regimen. At last available information, the titration was completed in 224 (75\%) patients, undergoing in $55(18 \%)$ and not completed in 21
(7\%). Titration duration was $7.3(1.0-25.7)$ weeks. The first maintenance dose achieved was 800 (50-2000) $\mu \mathrm{g}$ b.i.d and lasted for 15.7 (2.0-118.3) weeks. During the selexipag exposure period, $168(75 \%)$ patients remained on the same maintenance dose, $41(18 \%)$ patients had one dose adjustment and 15 (7\%) patients had $\geq 2$ dose adjustments.

With a median duration of 7.3 weeks, the observed titration period is in line with GRIPHON and the selexipag prescribing information. Approximately $25 \%$ of patients had further dose adjustments following titration.

Please refer to page A189 for declarations of interest related to this abstract.

\section{S80 THE DIAGNOSIS AND MANAGEMENT OF CATHETER- ASSOCIATED UPPER-EXTREMITY DEEP VENOUS THROMBOSIS (CA-UEDVT): A SYSTEMATIC REVIEW}

${ }^{1} \mathrm{O}$ Aniftos, ${ }^{2} \mathrm{~A}$ Scott, ${ }^{3} \mathrm{~A}$ Kafai Golahmadi, ${ }^{1} \mathrm{~A}$ Chapman. ${ }^{1}$ Ashford and St Peter's Hospital Trust, Chertsey, UK; ${ }^{2}$ St George's University of London, London, UK; ${ }^{3}$ School of Medicine, Imperial College London, London, UK

\subsection{6/thorax-2021-BTSabstracts.86}

Introduction and Objectives Central venous catheters are a main risk factor for deep venous thrombosis of the upper extremity (UEDVT), with $93 \%$ of all UEDVTs being catheter-

PRISMA 2009 Flow Diagram

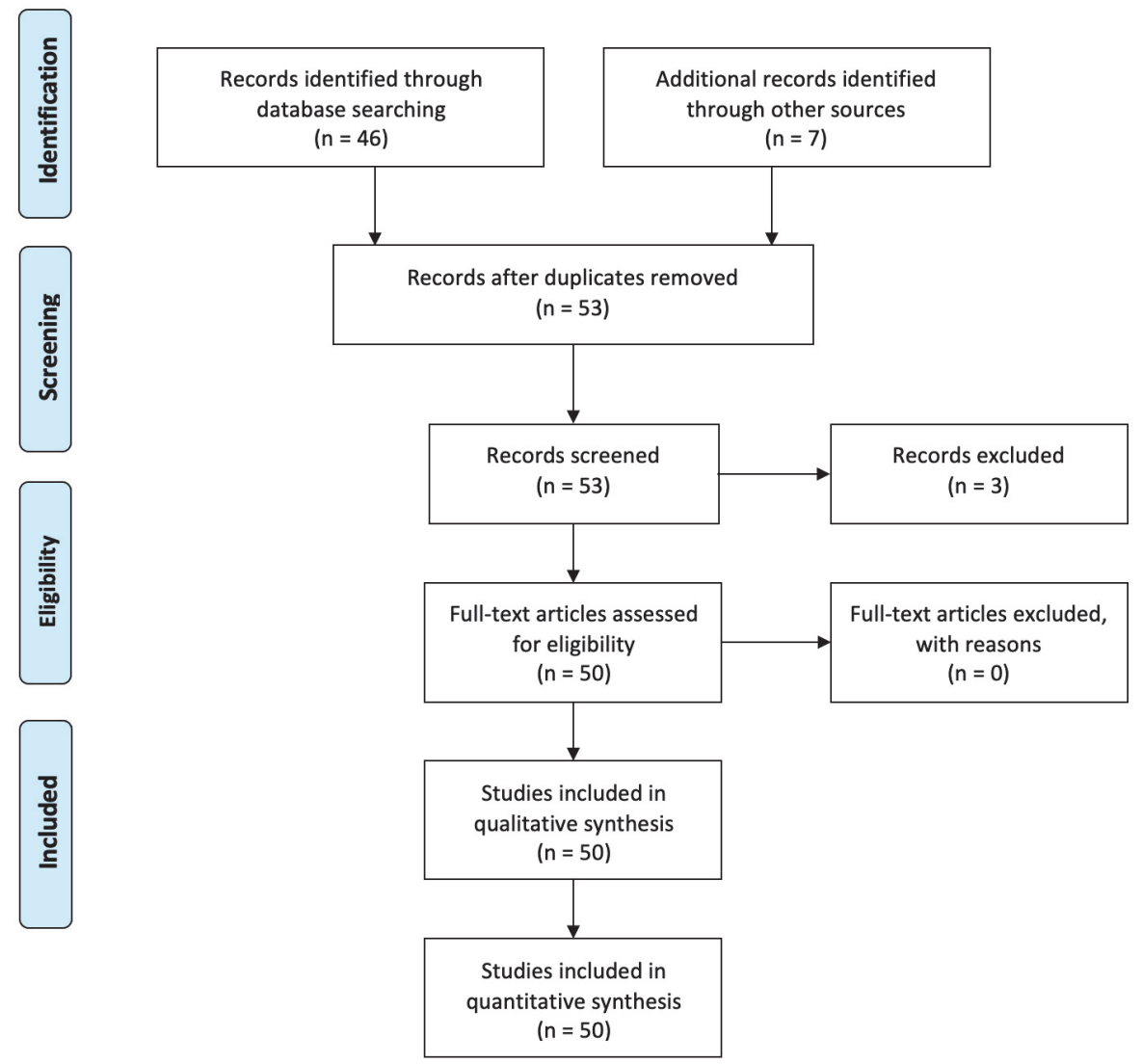


induced. Pulmonary embolism is an important sequela of DVT and albeit uncommon, remains a clinically significant cause of death with a mortality of up to 21.7 per 100,000 in the UK. In addition, $6 \%$ of pulmonary embolisms have an upper extremity source. We recognise the lack of official guidance on the diagnosis and management of CA-UEDVT. Herein, we aim to discuss the up-to-date management strategies for this prevalent condition.

Methods A systematic review was conducted according to PRISMA guidelines (search string: "catheter" AND "UEDVT") between 1997-2021 on PubMed. 53 manuscripts were screened by a single author (OA) on Rayyan and 50 were included in the final analysis. We extracted data on the institution, journal, citations, topic, sample size and outcomes.

Results Our results show that the commonest symptoms reported are ipsilateral upper extremity pain and/or discomfort, oedema, arm fatigue and discoloration at catheter entry site, but the majority of UEDVTs are asymptomatic. A high index of suspicion is therefore required.

For its diagnosis, venous duplex scan (VDS) is the commonest imaging modality used but it is less sensitive in paediatric patients and has limited use for central vasculature. In clinically suspected CA-UEDVT with negative VDS, contrastvenography - the gold-standard - can be used. It is, however, an invasive and technically complex procedure which requires use of contrast. For the management we recommend anticoagulation without removal of catheter for as long as catheter is in place and continued for at least three months after removal. (Low-molecular-weight-heparin in cancer patients and Direct-Oral-Anti-Coagulants in non-cancer patients unless contra-indicated). Consider removal if there is a catheter-associated infection, continuation of symptoms despite treatment and if the catheter is no longer functional or required. Finally, consider catheter-directed thrombolysis when indicated.

Conclusions Our findings provide a preliminary ground for further research into the diagnostic features of CA-UEDVT and may advise on the most up-to-date management algorithm for this condition.

\section{S81 10 YEAR RETROSPECTIVE AUDIT FROM AMBULATORY PULMONARY EMBOLISM PATHWAY IN A TERTIARY HOSPITAL}

R Sudhir, E Bailie, N Parmar. University Hospitals of Leicester NHS Trust, Leicestershire, UK

\subsection{6/thorax-2021-BTSabstracts.87}

Introduction The suspicion of PE (Pulmonary embolism) is a common reason for hospital admission and hospital stays. Being able to prevent admission in low risk PE patients has previously shown to be safe and cost effective with an established ambulatory service running in our hospital since 2010.

Methods A 10 year retrospective analysis from June 2010 to January 2020 was carried out using the PE database. Patients with suspected PE referred to the service using acceptance criteria (appendix a). PE risk was then stratified using the PE severity index (PESI). D-dimers were performed in the low and intermediate probability groups. Those with negative $\mathrm{d}$ dimers were discharged; those with high risk or positive ddimer underwent imaging in the form of CT pulmonary angiography (CTPA) or ventilation-perfusion (VQ) scanning. This was generally a same day service.
Results Total number of patients referred to the service was 6434. 2825 (43\%) were through bed bureau, $1491(23 \%)$ through Clinical decisions unit, 732 (11\%) though Emergency department and further $700(10.8 \%)$ through other services.

From the total number of 6434, 3724 (58\%) did not require any scans and were safely discharged from the service. CTPulmonary angiogram (CTPA) was performed in 2126 (33\%) and Ventilation-Perfusion (V/Q) scans in 584 (9\%). 2710 (42\%) patients underwent scanning with 429 (15.8\%) positive and $2281(84 \%)$ were negative. 6112 (95\%) of these patients were managed as outpatients. All patients were contacted by nurse led telephone follow-up, 1 week post diagnosis. Consultant led follow-up was variable, approximately $74 \%$ (321/429), due to multi-consultant service.

Conclusions Outpatient management of Pulmonary embolism is a safe and effective service.

\section{S82 USING CARDIAC MAGNETIC RESONANCE IMAGING TO ASSESS CARDIAC GEOMETRY IN THE DIAGNOSIS OF CHRONIC THROMBOEMBOLIC DISEASE AND CHRONIC THROMBOEMBOLIC PULMONARY HYPERTENSION}

${ }^{1} \mathrm{M}$ McGettrick, ${ }^{1} \mathrm{H}$ Dormand, ${ }^{1} \mathrm{M}$ Brewis, ${ }^{2} \mathrm{NN}$ Lang, ${ }^{1} \mathrm{M}$ Johnson, ${ }^{1} \mathrm{AC}$ Church. ${ }^{1}$ Scottish Pulmonary Vascular Unit, Glasgow, UK; ${ }^{2}$ University of Glasgow, Glasgow, UK

\subsection{6/thorax-2021-BTSabstracts.88}

Background Cardiac magnetic resonance (CMR) imaging is the gold standard tool for evaluating the right ventricle (RV) in chronic thromboembolic disease (CTED) and chronic thromboembolic pulmonary hypertension (CTEPH). Ventricular septal flattening, reflecting RV pressure overload in idiopathic pulmonary arterial hypertension (IPAH) has been quantified using the eccentricity index (EI) with echocardiography and pulmonary artery distensibility (PAD) has been shown to correlate with pulmonary artery pressure. These have not been evaluated for use in CTEPH using CMR. We assessed them in the detection of CTED and CTEPH and correlated with haemodynamics.

Methods CMR and right heart catheterisation were performed on 30 patients with CTEPH and 20 sex-matched controls without resting pulmonary hypertension (10 patients with no thrombotic disease and 10 with CTED) at a national pulmonary hypertension centre. Mid-papillary short axis view was used to assess the eccentricity index at end-systole and enddiastole. Main PAD was measured using velocity-encoded CMR, perpendicular to pulmonary artery.

Results EI at end-systole and end-diastole were significantly increased in CTEPH compared to controls (1.3 (0.5)vs1.0 (0.01); $\mathrm{p} \leq 0.01$ and (1.22 (0.2)vs0.98 (0.01); $\mathrm{p} \leq 0.01$, respectively). PAD was significantly reduced in CTEPH compared to controls $(0.13(0.1)$ vs $0.46(0.23) ; \mathrm{p} \leq 0.01)$. End-systolic EI and end-diastolic EI significantly correlated with pulmonary vascular haemodynamic indices, including mean pulmonary arterial pressure, cardiac output and with NTproBNP. End-systolic and End-diastolic EI correlated with exercise capacity as measured by 6-minute walk distance, and with pulmonary artery distensibility (R-value 0.8 ). Using ROC curves, an optimal threshold of 1.1 for both end-diastolic and end-systolic indices identified the presence of pulmonary hypertension. Both EI and PAD were able to differentiate the presence of CTED from normal. 